\title{
Chronic suppurative otitis media in Nepal: ethnicity does not determine whether disease is associated with cholesteatoma or not
}

\author{
D THORNTON ${ }^{1}$, T P C MARTIN ${ }^{2}, \mathrm{P} \mathrm{AMIN}^{1}, \mathrm{~S} \mathrm{HAQUE}^{3}, \mathrm{~S}_{\mathrm{WILSON}}^{4}, \mathrm{M} \mathrm{C} \mathrm{F} \mathrm{SMITH}^{5}$ \\ ${ }^{1}$ Birmingham University Medical School, ${ }^{2}$ Department of Otolaryngology, Queen Elizabeth Hospital, Birmingham, \\ ${ }^{3}$ Departments of Medical Statistics, ${ }^{4}$ Epidemiology, Birmingham University, and ${ }^{5}$ Department of Otolaryngology, \\ Hereford County Hospital and Worcester Royal Hospital, UK
}

\begin{abstract}
Objectives: To determine the frequency of cholesteatoma in a population of patients with chronic suppurative otitis media, and to determine whether this frequency is affected by ethnicity.

Patients: The study included 6005 patients with chronic suppurative otitis media seen during the course of 30 charitable surgical 'ear camps' in Nepal.

Main outcome measures: Proportion of patients with each subtype of disease, and their ethnicity. A secondary outcome measure was concordance of surname with Nepalese ethnic affiliation.

Results: A total of 762 patients were grouped as being of Tibeto-Mongolian origin, and 4875 as Indo-Caucasian. The rate of chronic suppurative otitis media with cholesteatoma, expressed as a proportion of the rate of all chronic suppurative otitis media subtypes, was 17.8 per cent in Tibeto-Mongolian patients and 18.6 per cent in IndoCaucasian patients $(p>0.05)$. The effect of other risk factors (i.e. age, gender and geographical district) on disease distribution was also non-significant. Analysis of secondary outcome measures indicated that patients' surnames were a reliable predictor of ethnicity in this Nepalese population.

Conclusion: There is almost complete concordance in proportions of patients with significant genetic, cultural, and even geographical heterogeneity, suggesting that, in Nepal, the aetiology of cholesteatoma owes little to these factors.
\end{abstract}

Key words: Ethnicity; Nepal; Otitis Media, Suppurative

\section{Introduction}

Chronic suppurative otitis media (CSOM), with or without cholesteatoma, is a significant cause of illhealth world-wide, with the majority of the burden of disease carried by the developing world. The World Health Organization (WHO) estimates that CSOM affects between 65 and 330 million individuals and causes 28000 deaths annually, with a disease burden of over 2 million disability-adjusted life years. ${ }^{1}$

Since 1993, the International Nepal Fellowship has provided charitable medical care in rural and impoverished regions of Nepal. ${ }^{2}$ This programme includes an otological service, which takes the form of two annual 'ear camps' in which out-patient medical and surgical services are provided to the local population of a Nepalese district over a 7-9 day period. Typically, some 1000 patients are seen in the outpatient clinic and some 100 otological surgical procedures performed, with the provision of approximately 100 hearing aids.
Traditionally, otologists have observed a distinction between CSOM associated with cholesteatoma and CSOM associated with tympanic membrane perforation. In this paper, we present data describing the distribution of these two types of CSOM in the TibetoMongolian and Indo-Caucasian populations of Nepal, among patients presenting to International Nepal Fellowship ear camps.

\section{Materials and methods}

\section{Data collection}

We retrieved routinely collected data relating to patients seen at 30 medical camps held throughout the three western regions of Nepal between 1995 and 2004 (see Figure 1). In broad terms, Nepalese people living in the northern districts, nearer the Tibetan plateau, are of Tibeto-Mongolian extraction (see Figure 2), while those in the more southern districts, nearer the Indian Gangetic plain, are of Indo-Caucasian 


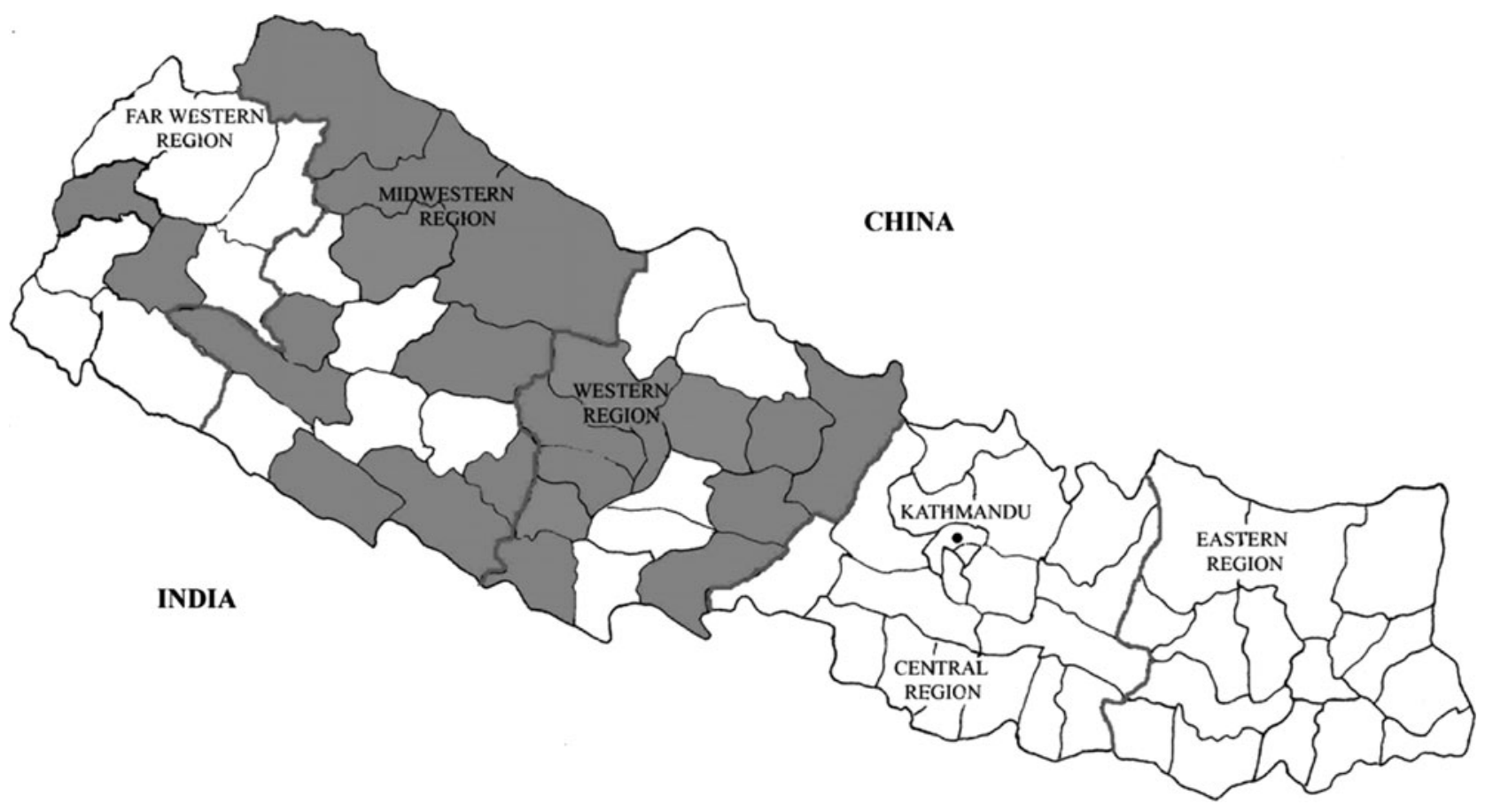

FIG. 1

Map of Nepal illustrating regions visited during the study period (shown in grey).

origin (see Figure 3). Although there has been much migration, racial, language and tribal affiliations are strong and marriages generally occur within ethnic groups. The geography of Nepal varies in a similar manner, from the high, mountainous Himalaya region in the northern strip to the low, flat Terrai strip along the Indian border.

The ear camps principally take place in the central hill regions, with some in both the higher mountains and the lower plains. Most regions have very steep hills and valleys; therefore, people's altitude and ambient temperature of residence are very variable, even for individuals living within a short distance of each other.

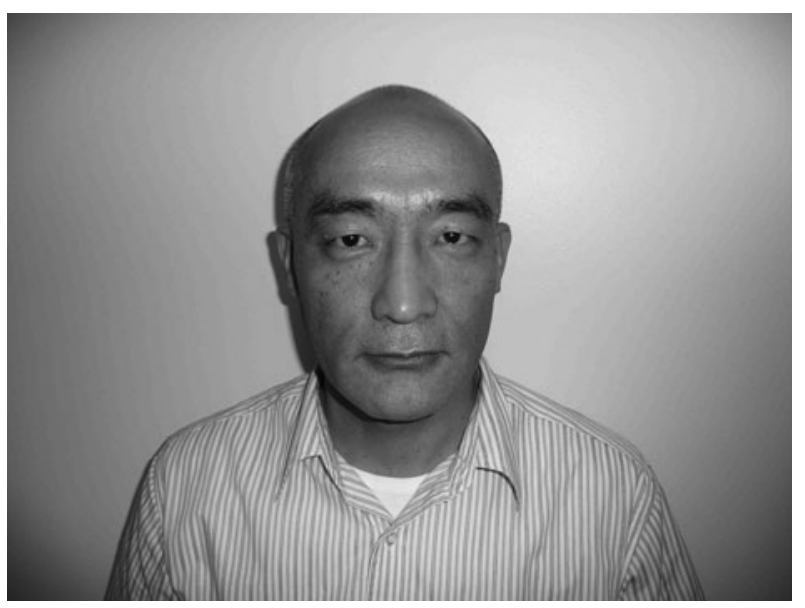

FIG. 2

Typical appearance of an Indo-Caucasian individual. Published with subject's permission.
Data were collected and recorded by volunteer ENT surgeons trained in the UK. Details of patient demographics, examination findings, diagnosis and treatment were entered into the Epi Info software package, developed by the Center for Disease Control and Prevention (sponsored by the WHO) and distributed at no cost to charitable organisations. ${ }^{3}$

All cases of CSOM were extracted from the database. With the assistance of a Nepali colleague working with the International Nepal Fellowship, these patients' ethnicity (Indo-Caucasian, TibetoMongolian or neither) was assigned based on their surname. Patients for whom no surname had been recorded were excluded from analysis, as were those

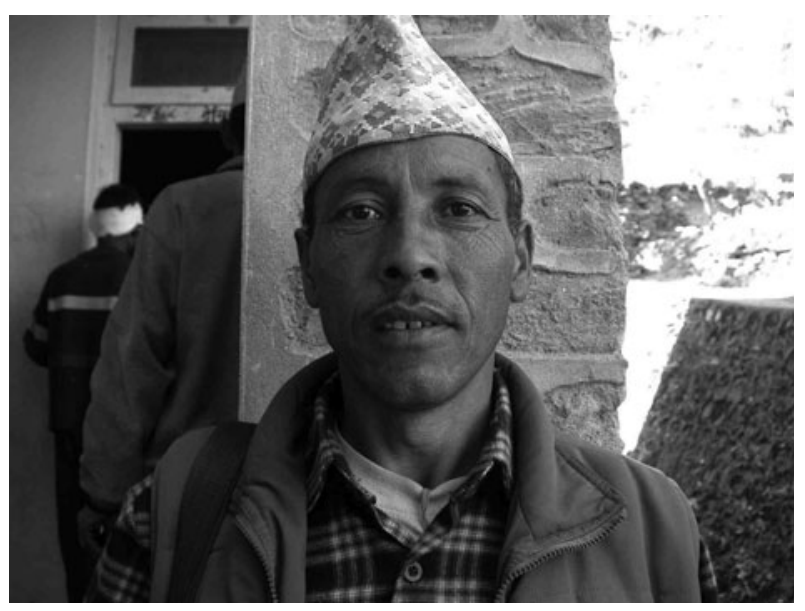

FIG. 3

Typical appearance of a Tibeto-Mongolian individual. Published with subject's permission. 
for whom the translator could not identify an ethnic grouping. Individuals with a diagnosis of either simple CSOM or CSOM with cholesteatoma were identified.

Patients in whom disease was bilateral and mixed were classified as having CSOM with cholesteatoma. Bilateral cases with one type of disease were counted once rather than twice. A separate analysis in which ears were counted individually did not lead to significantly different results.

\section{Subsidiary study}

The accuracy of a translator identifying ethnicity from surnames was assessed using an independent sample of over 100 people. This sample was opportunistically obtained from a medical camp set up by the International Nepal Fellowship in the Dailekh district of western Nepal and visited from 6 to 12 February 2008. Verbal, informed consent was obtained from participants with the help of a second, independent translator. Participants were asked to which ethnic group they attributed themselves. The same process of assigning ethnicity based on surname which had been used with the CSOM patients was then undertaken for these 100 individuals; the translator involved was blinded to the individuals' stated ethnicity.

\section{Statistical analysis}

An odds ratio, with confidence intervals, was used to compare the risk of one ethnic group demonstrating a disproportionate degree of either subtype of disease. A power analysis found that, in order to show a 10 per cent difference (i.e. 23 per cent versus 33 per cent, based on previously published studies), 4,5 giving an odds ratio of 1.65 , a sample of 452 people in each ethic group was required (90 per cent power, 5 per cent significance). The study easily achieved this power level. Binary logistic regression analysis was conducted to determine the factors (specifically ethnicity, age and gender) associated with a difference in the distribution of simple CSOM and CSOM with cholesteatoma.

In the subsidiary study, the proportion of correctly identified ethnicities from the sample was calculated. The 'gold standard' for this test was the ethnicity to which the participant assigned themselves. We then calculated the sensitivity, specificity and the positive predictive value of using surnames to identify ethnicity.

\section{Ethical approval}

Ethical approval for the study was granted by Birmingham University.

\section{Results}

\section{Chronic suppurative otitis media subtypes}

Six thousand and five cases of chronic suppurative otitis media were identified. Three hundred and sixtyeight (6 per cent) were excluded because no surname was recorded and ethnicity could thus not be identified. The patients' mean age was 19.1 years (standard deviation 14.2) and the age range was zero to 84 years. Forty per cent $(n=2241)$ of patients were female and 60 per cent $(n=3396)$ male. Patient characteristics are detailed in Table I.

A total of 5637 cases fulfilled both criteria, having a recorded surname and a diagnosis of CSOM. Of these cases, 4875 (86.5 per cent) were of Indo-Caucasian origin and 762 (13.5 per cent) of Tibeto-Mongolian origin. The proportion of CSOM with cholesteatoma was similar between the two groups (being 18.6 and 17.8 per cent, respectively; chi-square $=0.237$, one degree of freedom, $p=0.626$ ).

The odds ratio (Indo-Caucasian/Tibeto-Mongolian) for CSOM with cholesteatoma was 1.05 (95 per cent confidence interval (CI) 0.86-1.28). Logistic regression analysis did not identify any recorded sociodemographic factors (i.e. age, gender and district of residence) as being associated with the proportion of patients with simple CSOM versus CSOM with cholesteatoma.

\section{Subsidiary study}

The secondary aim of the study was to determine the accuracy of using surname to differentiate between Indo-Caucasian and Tibeto-Mongolian populations in Nepal. One hundred and nine individuals were recruited and provided their surname and ethnicity. Of these, 97 (89 per cent) described themselves as Indo-Caucasian and 12 (11 per cent) as TibetoMongolian. The translator was able to identify all of the Tibeto-Mongolian surnames, with no false positive results. However, there were seven false negative results, identifying Indo-Caucasian names as TibetoMongolian. This information is summarised in Table II. All the names identified within this subsidiary sample were also found within the list of diagnosed CSOM cases.

\begin{tabular}{|c|c|c|c|}
\hline PATIEN & $\begin{array}{r}\text { TABLE I } \\
\text { EMOGRAP }\end{array}$ & FEATURE & \\
\hline Parameter & $\mathrm{CSC}$ & & Total \\
\hline & Squamous & Mucosal & \\
\hline Ethnicity & & & \\
\hline Tibeto-Mongolian & 136 & 626 & 762 \\
\hline Indo-Caucasian & 906 & 3969 & 4875 \\
\hline Age range (y) & & & \\
\hline $1-15$ & 391 & 2498 & 2889 \\
\hline $16-25$ & 377 & 1057 & 1434 \\
\hline $26-35$ & 113 & 483 & 596 \\
\hline $36-45$ & 83 & 258 & 341 \\
\hline $46-59$ & 50 & 191 & 241 \\
\hline $60+$ & 28 & 108 & 136 \\
\hline Sex & & & \\
\hline Male & 622 & 2774 & 3396 \\
\hline Female & 420 & 1821 & 2241 \\
\hline
\end{tabular}




\begin{tabular}{lccr}
\multicolumn{4}{c}{ TABLE II } \\
SENSITIVITY AND SPECIFICITY OF SURNAME \\
ANALYSIS
\end{tabular}

Data represent patient numbers. *True ethnicity; ${ }^{\dagger}$ test ethnicity. Ethnic ID = identification of ethnicity

When sensitivity was taken as the proportion of correctly identified Indo-Caucasian names, the subsidiary study showed a sensitivity of 92.8 per cent ( 95 per cent CI 85-97). Specificity, taken as the proportion of correctly identified Tibeto-Mongolian names, was shown to be 100 per cent (95 per cent CI 70-100). The overall accuracy rate was 93.6 per cent (102 of 109 classified correctly). The most common mistake related to the classification of the surname 'Thapa', which was identified by our translator as TibetoMongolian; however, a number of patients with this surname identified themselves as Indo-Caucasian, suggesting that this surname was used in both ethnic groups.

In light of this result, we re-analysed the CSOM data, excluding all cases likely to have classification errors (i.e. those with the surname Thapa). Two hundred and seventeen cases were thus excluded from analysis; however, little difference was observed in the resultant estimated odds ratios for either CSOM with cholesteatoma (1.13 (95 per cent CI 0.89-1.43)). This sensitivity analysis of a modified data set confirmed our finding that there was no association between CSOM with cholesteatoma, or that of simple CSOM, and ethnicity.

\section{Discussion}

The role of genetics in the pathogenesis of chronic otitis media is disputed. It is well recognised that the condition is a disease of poverty most often found among socially deprived groups within a genetically homogeneous population. ${ }^{6}$ It has also been observed that deprived ethnic minorities often demonstrate high rates of chronic ear disease in general. It has been noted, for example, that New Zealand Maoris, Inuit peoples, native Americans and Australian Aboriginals all have high rates of chronic ear disease relative to the more affluent (and genetically distinct) dominant populations of their respective countries. ${ }^{7}$ What is not clear is whether this greater prevalence of disease among certain ethnic groups is due to genetic differences or to social deprivation.

There is an interesting body of otological research examining the association between ethnicity and CSOM subtype. In the 1970 s, Ratnesar ${ }^{8}$ studied a group of Caucasians, Inuit peoples and Algonkian Indians, and found cholesteatoma to be almost unknown in the latter two races. This study was supported by an assessment of eustachian tube patency, which found that Caucasians achieved less efficient mastoid and middle-ear cleft ventilation than the indigenous races, suggesting that this anatomical variation explained the observed pathological differences. Jassar et al. ${ }^{9}$ suggested that a low observed (although not quantified) rate of cholesteatoma among Aboriginal Australians could be explained by a posteriorly positioned incudo-stapedial apparatus, and hence a tendency for any retraction pocket to be self-cleansing.

Partly influenced by this work, our aim in the present study was to test an observational hypothesis (developed by the senior author, MCFS) that, in Nepal, cholesteatomatous disease was less common in Tibeto-Mongolian people than in Indo-Caucasian people. Interestingly, our study actually demonstrated a remarkable concordance between these two groups, calling into question the validity of ethnicity as a determinant of cholesteatoma pathogenesis, certainly within these two populations. Interestingly, in this large study, we did not observe the potential risk factors of age, sex or district (which could be a surrogate marker for altitude or social deprivation) to be associated with different rates of cholesteatomatous disease relative to mucosal chronic otitis media.

- Chronic otitis media is a well recognised disease of poverty

- Previous publications have suggested that the risk of cholesteatoma formation may vary for different ethnic groups

- This large study suggests that, in a Nepalese population seeking ENT care, patients with chronic suppurative otitis media with cholesteatoma represent approximately 20 per cent of all chronic suppurative otitis media cases

- In this population, ethnicity had no significant effect on whether chronic otitis media was cholesteatomatous or mucosal

Unfortunately, because our results come from a selfpresenting cohort rather than a population survey, we are unable to comment upon the prevalence of chronic otitis media in Nepal. However, other authors have reported significant levels of ear disease in this region. In a robust study, Little et al. ${ }^{4}$ calculated that 7.4 per cent of the Nepalese population had tympanic membrane pathology, comparing unfavourably with an estimated UK prevalence of chronic otitis media of 4.1 per cent. ${ }^{6}$ Interestingly, Little's group found a very similar rate of cholesteatomatous disease ( 24 per cent) to that seen in our study, and a similar value 
(22 per cent) was reported by Maharjan et al. ${ }^{5}$ in a study of schoolchildren in eastern Nepal.

In addition, we must acknowledge the risk of confounding factors in our study, due to the nature of the ear camps from which our data were drawn. Although there are very limited medical services (and particularly otological services) in the regions visited, there may have been a small proportion of relatively wealthy patients with ear disease who were able to access treatment by other means. Contrarily, there may also have been an unrepresented patient group who could not afford the sacrifice of time and resources required to visit an ear camp (which may have taken place a number of days' walk from their home and place of work).

Finally, although it is interesting to speculate on whether altitude influences middle-ear disease, and one might reasonably expect such an analysis in a paper with a geographical perspective in a country as famously mountainous as Nepal, such an analysis is unrealistic because of the diversity of terrain in the regions visited. Thus, individuals living in one region may live at very varying altitudes, and it would be inaccurate to categorise any region as 'high' or 'low' altitude.

\section{Subsidiary study}

Our subsidiary study was invaluable in enabling confident classification of the ethnicity of our population, and we hope that our results will be useful to other researchers in Nepal. Surname analysis is obviously a simplistic means of determining ethnicity, which has clear pitfalls and which will be variable in different groups. However, we would argue that, in Nepal (as in, for example, a Hispanic population in the US $)^{10}$ it is a useful research tool.

\section{Conclusion}

To some extent, the debate over whether CSOM is associated with cholesteatoma or not is less urgent now that it is well recognised that both variants (simple CSOM and CSOM with cholesteatoma) represent significant disease with potentially serious complications. ${ }^{11}$ Nevertheless, the pathogenesis of cholesteatoma is yet to be fully understood. This study, we argue, suggests that genetic factors may not be as significant as previously believed in determining whether an individual develops a cholesteatoma or a perforated eardrum.

\section{References}

1 Chronic suppurative otitis media: burden of illness and management options. Acuin J. jointly published by the Department of Child and Adolescent Health and Development and the Team for Prevention of Blindness and Deafness. World Health Organization, Geneva, 2004

2 International Nepal Fellowship. www.INF.org [4 Sept 2010]

3 CDC Epi Info ${ }^{\text {TM}}$. In: http://www.cdc.gov/Epiinfo/ [4 Sept 2010]

4 Little P, Bridges A, Guragain R, Frieman D, Prasad R, Weir N. Hearing impairment and ear pathology in Nepal. J Laryngol Otol 1993; 107:395-400

5 Maharjan M, Bhandari S, Singh I, Mishra SC. Prevalence of otitis media in school going children in Eastern Nepal. Kathmandu Univ Med J 2006;4:479-82

6 Browning GG, Gatehouse S. The prevalence of middle ear disease in the adult British population. Clin Otol 1992;17: 317-21

7 Miller ML. Epidemiology of otitis media: problem and research focus for geographers. Soc Sci Med 1979;13:233-6

8 Ratnesar P. Chronic ear disease along the coasts of Labrador and Northern Newfoundland. J Otol 1976;5:122-30

9 Jassar P, Murray P, Wabnitz D, Heidrich C. The posterior attic: an observational study of aboriginal Australians with chronic otitis media (COM) and a theory relating to the low incidence of cholesteatomatous otitis media versus the high rate of mucosal otitis media. Int J Pediatr Oto rhino laryngol 2006; 70:1165-7

10 Fiscella K, Fremont AM. Use of geocoding and surname analysis to estimate race and ethnicity. Health Serv Res 2006;41: 1482-500

11 Browning GG. The unsafeness of "safe" ears. J Laryngol Otol 1984;98:23-6

Address for correspondence:

Mr Thomas P C Martin,

20 Taylor Rd,

King's Heath,

Birmingham B13 0PG, UK

Fax: +44 (0)121 4241353

E-mail: tpcmartin001@gmail.com

Mr T P C Martin takes responsibility for the integrity of the content of the paper

Competing interests: None declared 